\title{
Fluorescein angiographic findings and clinical features in Fuchs' uveitis
}

\author{
Nadia Bouchenaki · Carl P. Herbort
}

Received: 15 October 2009/ Accepted: 29 March 2010/Published online: 18 April 2010

(C) Springer Science+Business Media B.V. 2010

\begin{abstract}
Fuchs' uveitis is very often diagnosed with substantial delay, which is at the origin of deleterious effects such as unnecessary treatment and its consequences. The aim of this study was to analyse the type and frequency of posterior inflammatory and fluorescein angiographic signs in Fuchs' uveitis in conjunction with other clinical signs. Patients seen at the Centre for Ophthalmic Specialised Care (COS) in Lausanne and the Memorial A. de Rothschild, Clinique Générale-Beaulieu in Geneva between 1995 and 2008 with the diagnosis of Fuchs' uveitis and who had undergone a fundus fluorescein angiography (FFA) were analysed. In addition to FFA signs, the data collected included age, gender, initial and final visual acuities, clinical findings at presentation, mean diagnostic delay and ocular complications. Between 1995 and 2008, 105 patients seen in our centres in Lausanne and Geneva were diagnosed with Fuchs' uveitis. Forty of them $(38.1 \%)$ had undergone at least one FFA. One
\end{abstract}

N. Bouchenaki · C. P. Herbort ( $\square)$

Inflammatory Eye Diseases, Centre for Ophthalmic

Specialised Care (COS), 6 Rue de la Grotte,

1003 Lausanne, Switzerland

e-mail: carl.herb@bluewin.ch

N. Bouchenaki · C. P. Herbort

Memorial A. de Rothschild, Clinique Générale-Beaulieu, Geneva, Switzerland

C. P. Herbort

University of Lausanne, Lausanne, Switzerland patient was excluded because of a concomittant diagnosis of multiple sclerosis. In 28 of 39 patients (71.2\%) diagnosis was not reached at presentation with a mean diagnosis delay of $3.67 \pm 4.86$ years (range: 1 month-24 years). The original erroneous diagnosis was intermediate uveitis in 16 patients (57.1\%), posterior uveitis in two patients $(7.1 \%)$, panuveitis in four patients (14.3\%) and anterior granulomatous uveitis in six patients $(21.4 \%)$. Fluorescein angiography demonstrated the presence of disc hyperfluorescence in 43/44 eyes (97.7\%), sectorial peripheral retinal vascular leaking in 6/44 eyes $(13.6 \%)$ and cystoid macular oedema in $4 / 44$ eyes $(9.1 \%)$, all of which were seen in eyes having undergone cataract surgery. Fuchs' uveitis was bilateral in 5/39 patients (12.8\%). The most frequent clinical signs were vitritis in $42 / 44$ eyes (95.5\%), stellate keratic precipitates in 41 eyes (93.2\%), posterior subcapsular opacities or cataract in 19 eyes (43.2\%), and heterochromia in 19 eyes (43.2\%). Fuchs' uveitis is a largely underdiagnosed uveitis, probably because the predominant vitreous involvement is ignored by many ophthalmologists. In addition, the nearly constant inflammatory fluorescein angiography findings reported here such as disc hyperfluorescence and, more rarely, peripheral retinal vascular leaking, are not well known and are not usually associated with Fuchs' uveitis but represent an additional factor leading to misdiagnosis. These findings need to be recognised in order to reduce diagnostic delay. 
Keywords Fuchs' uveitis · Vitritis ·

Fluorescein angiography · Disc hyperfluorescence ·

Diagnostic delay

\section{Background}

Fuchs' uveitis is a particular type of uveitis described comprehensively for the first time in 1906 by Ernst Fuchs [1]. In the 15th edition of his textbook Fuchs described the main features that are crucial in diagnosing Fuchs' uveitis: these include fine retrodescemetic keratic precipitates (KPs), vitreous infiltration, absence of iris inflammation, atrophic iris changes, lens opacification and good tolerance to surgery [2]. Fuchs' uveitis is seen around the world [3-6] and its frequency in uveitis studies varies from $2 \%$ in Japan [7] and Portugal [8] to $8.32 \%$ in Italy [9]. Its aetiology is unknown but the main hypothesis is that an infectious triggering factor, possibly rubella virus and/or other viruses [10-12], is at the origin of an autoimmune disease in genetically susceptible patients [13-15]. In most instances the condition is benign and does not require any anti-inflammatory therapy [16]. Although vitreous changes were clearly identified by Fuchs as a major sign in this condition along with typical KPs, iris atrophy and lens opacification, most of the attention has been given to the anterior clinical signs and vitreous involvement has been neglected in many studies over the years [1315, 17-20]. Although vitritis is cited in most studies, it does not usually appear in the abstracts of these articles nor is it put forward as a major element and is therefore not perceived by the average clinician to be characteristic of the condition. The presence of vitritis seems to be at the origin of misdiagnosis or of diagnostic delay.

Fluorescein was used to study Fuchs' uveitis for the first time in 1946 by Amsler who demonstrated increased penetration of fluorescein from the blood circulation into the anterior chamber corresponding to increased permeability of the blood-aqueous barrier [21]. This was later confirmed by fluorescein iris angiography showing leaking iris vessels around the pupil [22, 23]. Norrsell et al. found increased aqueous laser flare values and leaking iris vessels on fluorescein angiography in Fuchs' uveitis compared to normal eyes, postulating that leaking iris vessels are probably at the origin of the increased flare, an indication of an inflammatory process [24]. Less interest has been devoted to fundus fluorescein angiography (FFA) in Fuchs' uveitis except for a recent review of clinical features in Chinese Fuchs' uveitis patients which mentioned the presence of disc staining and retinal capillary leakage [25]. To the best of our knowledge this is, however, the first study specifically aimed at reporting FFA findings in Fuchs' uveitis.

\section{Patients and methods}

Charts of patients seen at the Centre for Ophthalmic Specialised Care (COS) in Lausanne and at Memorial A. de Rothschild Clinique Générale-Beaulieu in Geneva, Switzerland between 1995 and 2008 were reviewed. Patients with the diagnosis of Fuchs' uveitis who had undergone at least one fluorescein angiography were identified and their cases were studied retrospectively. All uveitis patients routinely have a complete uveitis ophthalmologic work-up. A detailed uveitis history is obtained including, especially in cases of Fuchs' uveitis, the onset of disease, the wrong diagnoses that were made and the treatments received. A complete ocular examination is performed that comprises laser flare photometry and gonioscopy, in addition to routine features such as Snellen visual acuity, slit-lamp examination, aplanation tonometry and funduscopy in mydriasis. Fluorescein angiography had already been performed before we saw the patients or was performed to ascertain the diagnosis by showing absence of cystoid macular oedema (CMO).

Particular attention was given to the signs found in Fuchs' uveitis such as laterality, presence and disposition of KPs, heterochromia, iris structure, presence of Koeppe nodules, presence of abnormal vessels in the irido-corneal angle, presence of opacities in the crystalline lens, vitreous infiltration and fundus lesions or scars.

\section{Results}

General findings and epidemiological data

Between 1995 and 2008, 105 patients (53 men and 52 women) seen in the uveitis clinic at the Centre for 
Table 1 Clinical characteristics of the study population

\begin{tabular}{ll}
\hline Number of patients & 39 \\
Age (years, range) & $39(11-71)$ \\
Gender & 20 \\
Male & 19 \\
Female & $3.6(0-24)$ \\
Duration of uveitis before diagnosis (years, range) & \\
Laterality & 34 \\
Unilateral & 5 \\
Bilateral & \\
\hline
\end{tabular}

Ophthalmic Specialised Care (COS) in Lausanne and at the Memorial A. de Rothschild Clinique GénéraleBeaulieu in Geneva were diagnosed with Fuchs' uveitis. The proportion of referred patients was $86 \%$. Forty patients $(38.1 \%)$ had a fluorescein angiography performed. One patient was excluded because the diagnosis of multiple sclerosis was established during the follow-up. There were 20 male and 19 female patients, and the mean age at diagnosis was 39 years (range: 11-71 years). In five of 39 patients (12.8\%), involvement was bilateral giving a total of 44 Fuchs' eyes included in the study. In 28 of 39 patients (71.2\%) diagnosis was not reached within 1 month after presentation to the ophthalmologist and the mean duration of the diagnostic delay was $3.67 \pm 4.86$ years (1 month-24 years) (Table 1 ). The most frequent erroneous diagnoses were intermediate uveitis in 16 of 28 patients $(57.1 \%)$, posterior uveitis in two patients $(7.1 \%)$ and panuveitis in four patients $(14.3 \%)$ making a total of 22 of 28 patients (78.6\%) where Fuchs' uveitis was mistaken for a uveitis of the posterior segment. In six patients $(21.4 \%)$ the improper diagnosis was anterior granulomatous uveitis.

Fluorescein angiographic findings (Table 2)

In 39 patients fluorescein angiography had been performed at some time during evolution. Taking into account the five bilateral cases, a total of 44 eyes had an FFA performed that could be included in this study.

Disc hyperfluorescence was present in all but one patient (eye) (97.7\%). Disc staining was evaluated on the late frame of angiography and graded as light, moderate or severe according to the intensity of the fluorescence (Fig. 1). The only case without disc hyperfluorescence was known to have post-traumatic
Table 2 Fluorescein angiographic findings (44 eyes)

\begin{tabular}{llr}
\hline & $\begin{array}{l}\text { Number of } \\
\text { cases }\end{array}$ & $\%$ \\
\hline Disc hyperfluorescence & 43 & 97.7 \\
Slight & 26 & 60.5 \\
Moderate & 8 & 18.6 \\
Severe & 9 & 20.9 \\
Mid-peripheral retinal vascular leaking & 6 & 13.6 \\
Cystoid macular oedema (post-cataract & 4 & 9.1 \\
surgery) & & \\
\hline
\end{tabular}

disc atrophy previous to the diagnosis of Fuchs' uveitis (Fig. 2). Nine eyes (20.9\%) presented a severe disc hyperfluorescence, eight eyes presented a moderate disc hyperfluorescence (18.6\%) and 26 eyes presented a slight disc hyperfluorescence $(60.5 \%)$. Interestingly, the severity of disc hyperfluorescence was not correlated with the degree of vitreous infiltration. Furthermore, in patients who underwent more than one fluorescein angiography during follow-up, disc staining remained mostly unchanged on successive fluorescein angiographies (Fig. 3).

In the five patients with bilateral involvement, there was an equal vitreous infiltration between the two eyes, ranging from 0.5 to $3+$ cells. In all patients, a symmetrical bilateral disc hyperfluorescence was present (Fig. 4).

A slight mid-peripheral retinal vascular leakage was present in six eyes (13.6\%) (Fig. 5). A chorioretinal scar was seen at the fundus examination in five eyes $(11.4 \%)$, but never presented as a pathological hyperfluorescence on fluorescein angiography.

Four patients $(9.1 \%)$ showed macular oedema, and all of them had previously undergone cataract surgery (Fig. 6). None of the non-operated patients showed CMO and absence of angiographic macular oedema in longstanding uveitis with pronounced vitreous infiltration was considered confirmatory of the diagnosis of Fuchs' uveitis.

Clinical symptoms and signs

The most common symptoms reported were the presence of floaters in 20 patients $(51.3 \%)$, decreased vision in 15 patients $(38.5 \%)$ and ocular pain or discomfort in three patients $(7.7 \%)$.

The clinical signs are listed in Table 3 . The most frequent sign found, vitreous infiltration, was present 
Fig. 1 a A 21-year-old man with typical Fuchs' KPs, heterochromia, vitreous cells $2+$, left disc hyperhaemia, and hyperfluorescent optic disc. b Angiographic sequence showing progressive disc staining
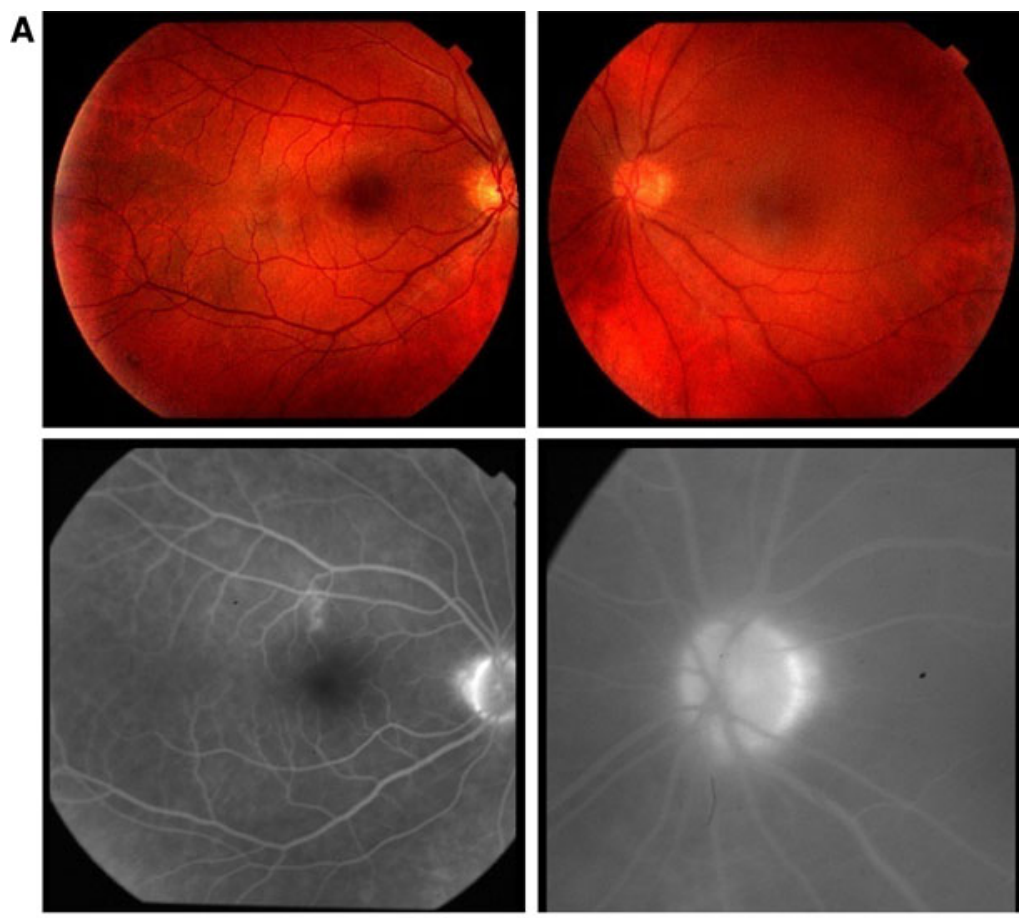

B
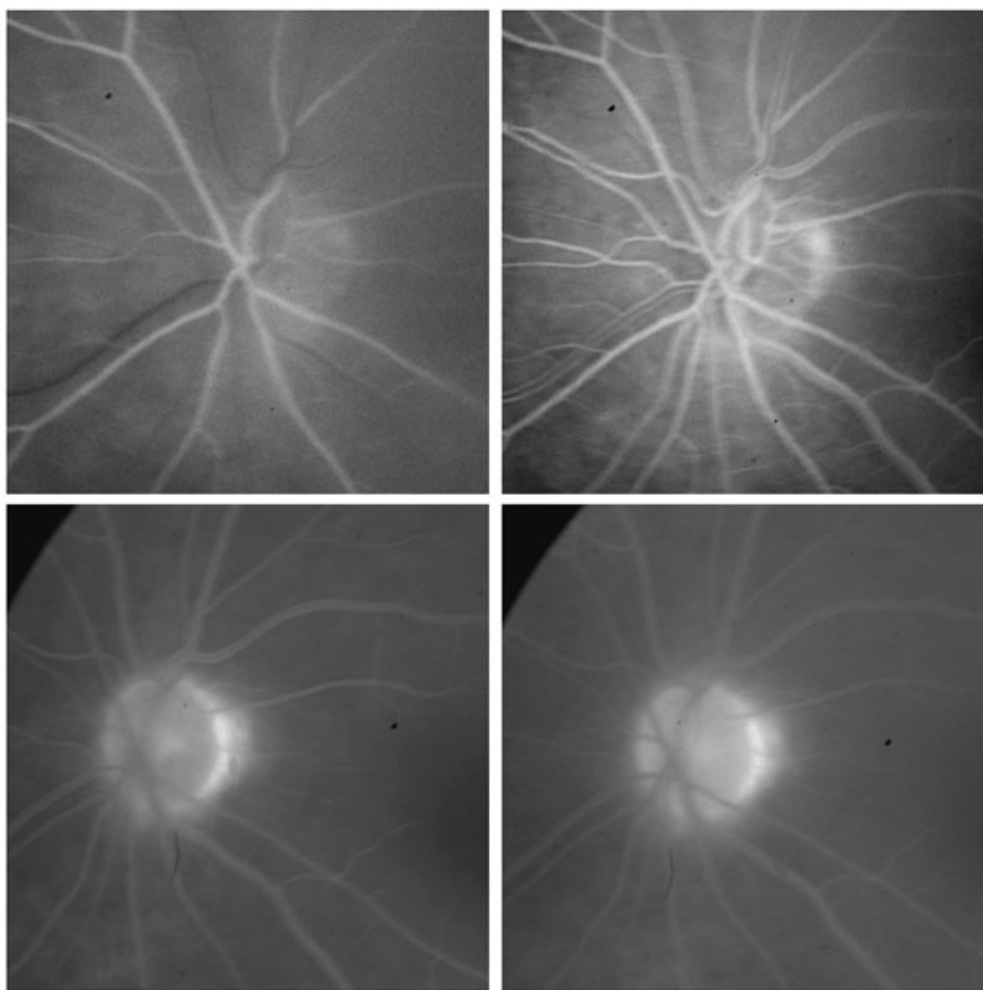

in 42 of 44 eyes $(95.5 \%)$. The characteristic stellate KPs were present in 41 of 44 eyes (93.2\%). Opacities of the crystalline lens, mostly posterior subcapsular opacification, were present in 19 eyes $(43.2 \%)$. Ten patients had undergone cataract surgery; the operation was performed in six of these patients before the 
Fig. 2 A 64-year-old female with partial traumatic optic nerve avulsion with Fuchs' uveitis. Lack of disc hyperfluorescence probably due to optic nerve atrophy

Fig. 3 Persistence of disc hyperfluorescence on successive fluorescein angiographies, 2 years apart, in a 50-year-old female with typical KPs, heterochromia and vitreous cells $3+$. First angiography: two pictures on the left; second angiography: two pictures on the right
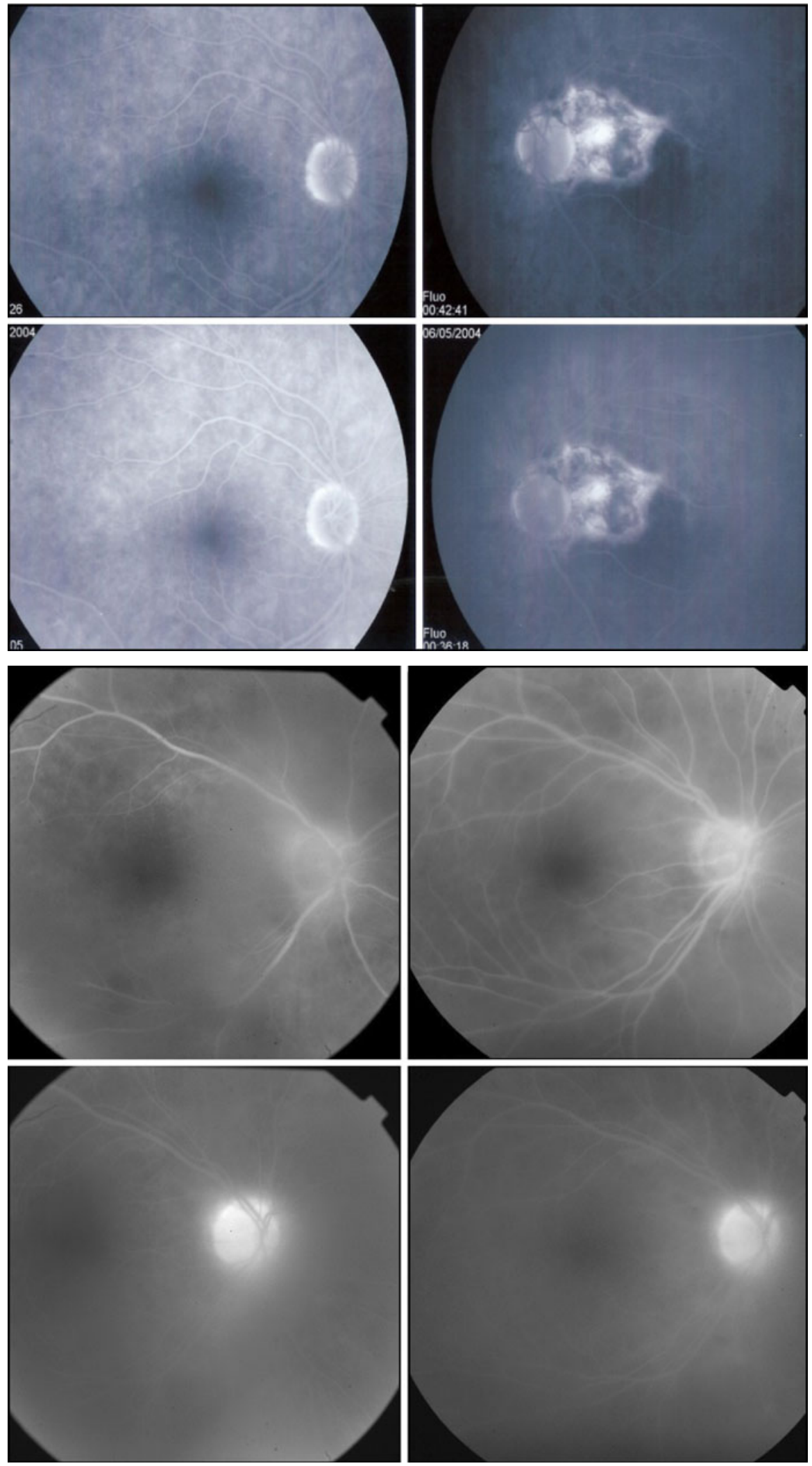


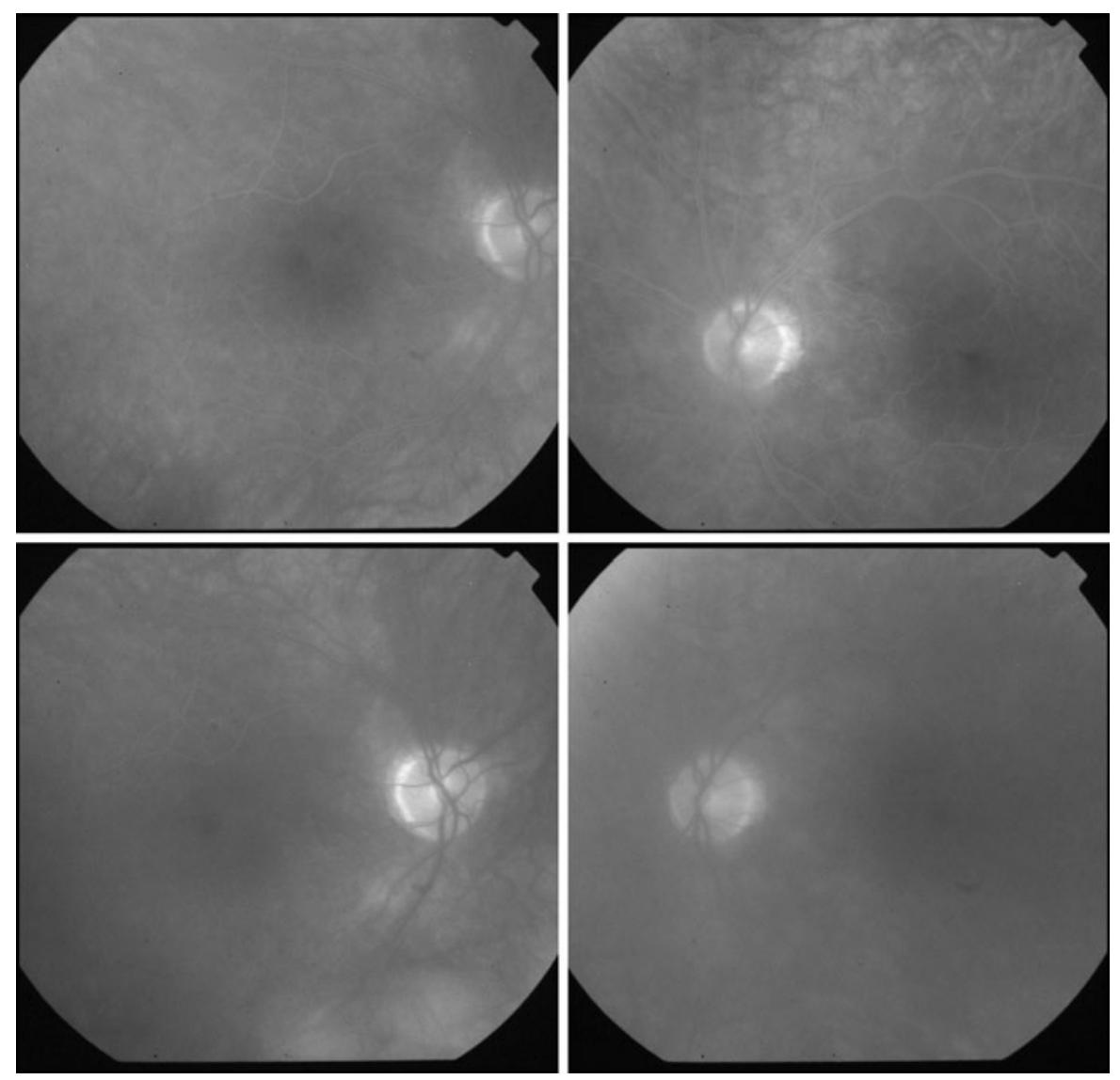

Fig. 4 Symmetrical disc hyperfluorescence in a bilateral Fuchs' uveitis case presenting typical KPs, vitreous cells of +0.5 and no heterochromia

Fig. 5 Peripheral vascular leakage in a young female showing typical KPs, heterochromia, cataract and vitreous cells $1+$
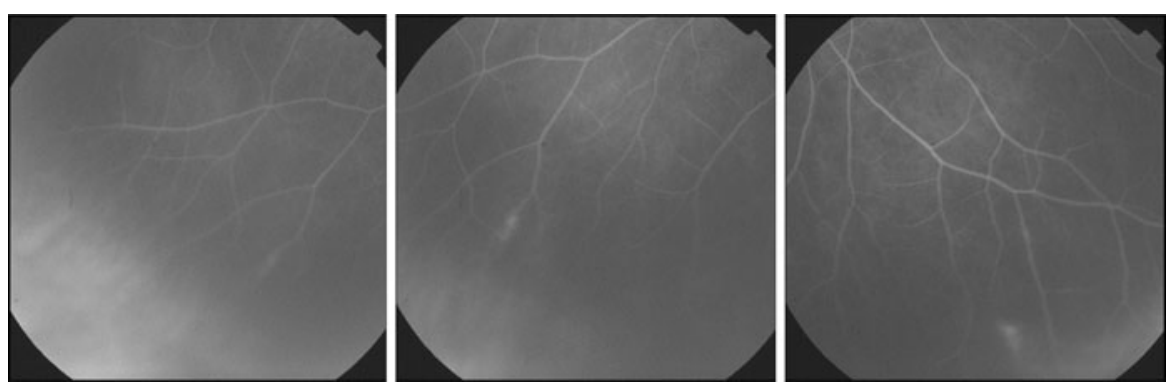

diagnosis of Fuchs' uveitis was made. Iris atrophy or heterochromia was found in only 19 eyes (43.2\%). Koeppe nodules were seen in three eyes $(6.8 \%)$ and abnormal vessels in the irido-corneal angle were seen in five eyes $(11.4 \%)$. None of the eyes examined showed posterior synechiae. The mean intraocular pressure at presentation was $13.8 \pm 4.95 \mathrm{mmHg}$. Eight eyes (18.2\%) presented with either hypertension or glaucoma and only two needed glaucoma surgery. The breakdown of the blood-aqueous barrier, measured by laser flare photometry, was minimal, amounting to $8.62 \pm 4.2 \mathrm{ph} / \mathrm{ms}$ at presentation and remaining stable at $8.45 \pm 4.3 \mathrm{ph} / \mathrm{ms}$ at the end of follow-up.

Fundus examination was unremarkable in 19 eyes (43.2\%) and difficult to visualise in seven eyes due to the presence of a vitreous haze. A disc hyperhaemia or blurred disc margins were present in 12 eyes 


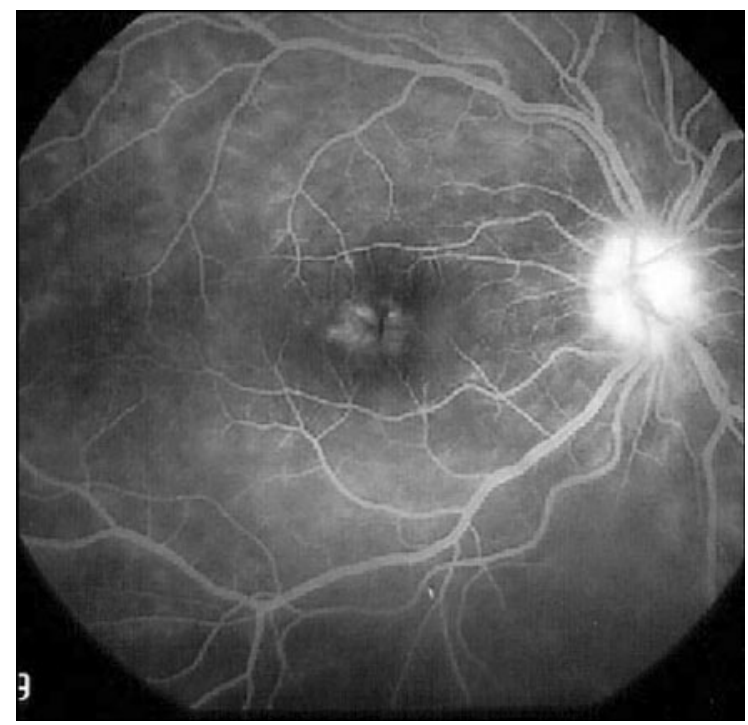

Fig. 6 Cystoid macular oedema after cataract surgery

Table 3 Clinical findings of the study population (44 eyes)

\begin{tabular}{lrr}
\hline & Number of cases & $\%$ \\
\hline Vitreous infiltration & 42 & 95.5 \\
Characteristic KPs & 41 & 93.2 \\
Iris atrophy/heterochromia & 19 & 43.2 \\
Crystalline lens opacities & 19 & 43.2 \\
Irido-corneal angle abnormal vessels & 5 & 11.4 \\
Koeppe nodules & 3 & 6.8 \\
Papillary hyperhaemia or blurred disc & 12 & 27.3 \\
Chorioretinal scar & 5 & 11.4 \\
Peripheral hole or tear & 3 & 6.8 \\
Cystoid macular oedema & 0 & 0 \\
on funduscopy & & \\
\hline
\end{tabular}

(27.3\%). A chorioretinal scar was seen in five eyes (11.4\%), either peripheral (three eyes) or central (macular in one eye, parapapillary in one eye). Three patients $(6.8 \%)$ developed a peripheral hole or tear that had to be sealed off by laser therapy. CMO was not seen by funduscopic examination in any of the patients.

\section{Discussion}

To the best of our knowledge this is the first study aimed at systematically analysing FFA findings in Fuchs' uveitis. To date one article from China mentioned inflammatory angiographic signs in their Fuchs' series [25]. Our study is the result of chance findings resulting from the analysis of fluorescein angiographies brought by referred patients. In suspected cases of Fuchs' uveitis, additional angiographies were performed when a blurred or hyperhaemic disc was present at fundoscopy or, at the time where we did not have an OCT instrument at our disposal, to show the absence of CMO despite prolonged vitreous inflammation, so giving an additional element in confirming Fuchs' uveitis. FFA was also performed in cases with a confirmed diagnosis of Fuchs' uveitis that showed a decrease of visual acuity after cataract surgery. The first remarkable finding was the quasiconstant involvement of the optic disc in Fuchs' uveitis always showing hyperfluorescence of diverse grade, except in one case where the optic disc had been devascularised by blunt trauma causing partial disc avulsion in the past. The rate of almost $100 \%$ disc hyperfluorescence might be a result biased towards hyperpositivity because, in a large proportion of cases, angiographies that had already been performed during previous consultations elsewhere were included, with the possible corollary that only patients with more severe inflammation had undergone an FFA. Second, retinal vasculitis of the small peripheral retinal vessels is not a rare occurrence (present in 13.6\%). Third, none of the Fuchs' patients presented CMO despite frequent severe and prolonged vitreous infiltration and prominent hot discs. This finding is so consistent that it should be used as an additional diagnostic criterium for Fuchs' uveitis. The exception to this are Fuchs' patients that have undergone cataract surgery and it should be noted that once an eye with Fuchs' uveitis has been operated on, the macula is no longer protected from the development of CMO, representing the fourth important finding of our study.

When looking at the erroneous diagnoses before the diagnosis of Fuchs' uveitis was made, uveitis of the posterior segment made up the majority of wrong diagnoses in over $70 \%$ of the non-diagnosed patients. It appears that the vitreous involvement is not identified as being associated with Fuchs' uveitis and therefore diverts the clinician from the diagnosis of Fuchs' uveitis. This misorientation can be additionally strengthened and the clinician further misled by the presence of disc hyperfluorescence and the less frequent peripheral retinal vasculitis on fluorescein angiography. In the literature of the last 2-3 decades, 
Fuchs' uveitis is mostly identified as an anterior uveitis, and it is the anterior involvement that is described and analysed in detail leaving posterior segment involvement in the background, although it is cited in most of the papers [13, 15, 17-20].

The hypothetical mechanisms to explain disc hyperfluorescence on fluorescein angiography in Fuchs' uveitis could be either an inflammatory breakdown of the blood-ocular barrier, similarly to the blood-aqueous barrier breakdown shown in iris fluorescein angiography studies [21, 23, 24], or the occurrence of mechanical traction by heavy infiltrated vitreous around the optic disc (and along peripheral retinal vessels, explaining vascular retinal leaking). However, it should be noted that optic disc hyperfluorescence is not proportional to vitreous infiltration and pronounced disc hyperfluorescence can be found even in a very slightly infiltrated vitreous.

Given the large number of misdiagnoses of Fuchs' uveitis due to unawareness of posterior segment involvement, it is crucial to incorporate these posterior signs into the main clinical picture of Fuchs' uveitis, as mentioned by Fuchs himself [1]. Inflammatory angiographic signs are now known to be part of posterior involvement that should no longer mislead the clinician and allow diagnosis as early as possible in order to avoid unnecessary work-up, but more importantly to avoid useless, potentially dangerous therapy.

In summary, the heterogeneity of the clinical presentation of Fuchs' uveitis renders the diagnosis difficult in some patients, especially when posterior features are prominent and not recognised as being part of the clinical picture of Fuchs' uveitis. Therefore, in order to avoid misdiagnosis in future, recognition of vitreous involvement and angiographic inflammatory signs as being characteristic signs of Fuchs' uveitis is important. Beside the usual wellknown features such as characteristic KPs, iris structure changes, heterochromia, lens opacification, Koeppe nodules and the constant absence of posterior synechiae, the less well-known posterior features presented here should be taken into account in the diagnosis of Fuchs' uveitis. This should include angiographic findings reported in the present study such as the absence of CMO despite pronounced and prolonged inflammatory involvement in non-operated Fuchs' eyes and disc hyperfluorescence, both of which are present in almost $100 \%$ of patients.

\section{References}

1. Fuchs E (1906) Ueber Komplikationen der Heterochromie. Z Augenheilkd 15:191-212

2. Fuchs E (1926) Aetiologie und Formen der Iridozyklitis. In: Fuchs E (ed) Lehrbuch der Augenheilkunde. Franz Deuticke, Leipzig und Wien, pp 463-471

3. Tran VT, Auer C, Guex-Crosier Y, Pittet N, Herbort CP (1994) Epidemiology of uveitis in Switzerland. Ocul Immunol Inflamm 2:169-176

4. Kilstra A, Rothova A, Baarsma GS, Zaal MJM, Fortuin ME, Schweitzer C, Glasius E, de Jong PT (1987) Computer registration of uveitis patients. Doc Ophthalmol 67:139143

5. Soheilan M, Heidari K, Yazdani S, Shahsavari M, Ahmadieh H, Dehghan MH (2004) Patterns of uveitis in a tertiary eye care center in Iran. Ocul Immunol Inflamm 12:297-310

6. Yang P, Zhang Z, Zhou H, Li B, Huang X, Gao Y, Zhu L, Ren Y, Klooster J, Kijsltra A (2005) Clinical patterns and characteristics of uveitis in a tertiary center for uveitis in China. Curr Eye Res 30:943-948

7. Higuchi M, Ohno S, Matsuda H (1982) Clinical characteristics of Fuchs' heterochromic iridocyclitis. Rinsho Ganka 36:1275-1280

8. Palmares J, Couthino MF, Castro-Correira J (1990) Uveitis in northern Portugal. Curr Eye Res 27(suppl):31-34

9. Pivetti-Pezzi P, Accorinti M, La Cava M, Collobelli Gisoldi RA, Abdulaziz MA (1996) Endogenous uveitis: an analysis of 1417 cases. Ophthalmologica 210:234-238

10. Quentin C, Reiber H (2004) Fuchs heterochromic cyclitis: rubella virus antibodies and genome in aqueous humor. Am J Ophthalmol 138:46-54

11. De Groot-Mijnes JDF, De Visser L, Rothova A, Schuller M, Van Loon AM, Weersink AJL (2006) Rubella virus is associated with Fuchs heterochromic iridocyclitis. Am J Ophthalmol 141:212-214

12. Chee SP, Bacsal K, Jap A, Se-Thoe SY, Chang CL, Tan BH (2008) Clinical features of cytomegalovirus anterior uveitis in immunocompetent patients. Am J Ophthalmol 145(5):834-840

13. La Hey E, Baarsma GS, De Vries J, Kijlstra A (1991) Clinical analysis of Fuchs' heterochromic cyclitis. Doc Ophthalmol 78:225-235

14. Liesegang TJ (1982) Clinical features and prognosis in Fuchs' uveitis syndrome. Arch Ophthalmol 100:1622-1626

15. Jones NP (1991) Fuchs' heterochromic uveitis. A reappraisal of the clinical spectrum. Eye 5:649-661

16. Schwab IR (1990) Fuchs' heterochromic iridocyclitis. Int Ophthalmol Clin 30:252-256

17. Fearnley IR, Rosenthal AR (1995) Fuchs' heterochromic iridocyclitis revisited. Acta Ophthalmol Scand 73:166-170

18. Higuchi M, Ohno S, Matsuda H (1982) Clinical features of heterochromic cyclitis (Fuchs) in Japan. Jpn Clin Ophthalmol 36:1275-1280

19. Tabbut BR, Tessler HH, Williams D (1988) Fuchs' heterochromic iridocyclitis in blacks. Arch Ophthalmol 106:1688-1690

20. Velilla S, Dios E, Herreras JM, Calonge M (2001) Fuchs' heterochromic iridocyclitis: a review of 26 cases. Ocul Immunol Inflamm 9:169-175 
21. Amsler M, Huber A (1946) Methodik und erste klinische Ergebnisse einer Funktionsprüfung der Blut-Kammerwasser-Schranke. Ophthalmologica 111:155-176

22. Loewenfeld IE, Thompson HS (1973) Fuchs's heterochromic cyclitis: a critical review of the literature. 1. Clinical characteristics of the syndrome. Surv Ophthalmol 17:394-457

23. Saari M, Vuorre I, Nieminen H (1978) Fuchs's heterochromic cyclitis: a simultaneous bilateral fluorescein angiographic study of the iris. Br J Ophthalmol 62:715721

24. Norrsell K, Holmer AK, Jacobson H (1998) Aqueous flare in patients with monocular iris atrophy and uveitis. A laser flare and iris angiography study. Acta Ophthalmol Scand 76:405-412

25. Yang P, Fang W, Jin H, Li B, Chen X, Kijlstra A (2006) Clinical features of Chinese patients with Fuchs' syndrome. Ophthalmology 113:473-480 